Published in final edited form as:

J Am Acad Orthop Surg. 2012 October ; 20(10): 668-669. doi:10.5435/JAAOS-20-10-668.

\title{
Sex Differences in Osteoarthritis of the Knee
}

\author{
Dr. Barbara D. Boyan, PhD, \\ Wallace H. Coulter Department of Biomedical Engineering, College of Engineering, Georgia \\ Institute of Technology, Atlanta, GA \\ Dr. Laura Tosi, MD, \\ Children's Research Institute, the Department of Orthopaedic Surgery and Sports Medicine, \\ George Washington University, Washington, DC
}

Dr. Richard Coutts, MD,

University of California San Diego Medical Center, San Diego, CA

Dr. Roger Enoka, PhD,

Department of Integrative Physiology, University of Colorado, Boulder, CO

Dr. David A. Hart, PhD,

Department of Microbiology, Immunology, and Infectious Diseases, the University of Calgary,

Calgary, Alberta, Canada

Dr. Daniel P. Nicolella, PhD, Mechanics and Materials Section, Southwest Research Institute, San Antonio, TX

Dr. Karen Berkley, PhD,

Program in Neuroscience, Department of Psychology, Florida State University, Tallahassee, FL

Dr. Kathleen Sluka, PT, PhD,

Department of Physical Therapy and Rehabilitation Science, the University of lowa, lowa City, IA

Dr. Kent Kwoh, MD,

Dr. Boyan or an immediate family member is a member of a speakers' bureau or has made paid presentations on behalf of Titan Spine; serves as a paid consultant to Exactech, the Musculoskeletal Transplant Foundation, the National Institutes of Health (NIAMS \& NICHD), NuVasive, Degradable Solutions AG, Titan Spine, Spineology, and Alacer, and as an unpaid consultant to Institut Straumann AG; has stock or stock options held in MedShape Solutions, ArthroCare, Carticept Medical, and SpherIngenics; has received research or institutional support from the Musculoskeletal Transplant Foundation; and serves as a board member, owner, officer, or committee member of the International Conferences on the Chemistry and Biology of Mineralized Tissue, the American Association for the Advancement of Science, and the American Association for Dental Research. Dr. Tosi or an immediate family member serves as a paid consultant to Amgen, KCI, and Medtronic and serves as a board member, owner, officer, or committee member of the American Academy for Cerebral Palsy and Developmental Medicine, American Orthopaedic Association, and Society for Women's Health Research Medical Society of the District of Columbia. Dr. Coutts or an immediate family member serves as a paid consultant to Exactech. Dr. Enoka or an immediate family member serves as a paid consultant to Cytokinetics. Dr. Nicolella or an immediate family member serves as a paid consultant to Merck. Dr. Berkley or an immediate family member has stock or stock options held in Eli Lilly, Johnson \& Johnson, Procter \& Gamble, and Sanofi-Aventis and serves as a board member, owner, officer, or committee member of the Organization for the Study of Sex Differences (OSSD). Dr. Sluka or an immediate family member has received research or institutional support from Medtronic. Dr. Kwoh or an immediate family member serves as a paid consultant to Pfizer and Novartis. Dr. O'Connor or an immediate family member serves as a paid consultant to Stryker and Zimmer, serves as an unpaid consultant to and has stock or stock options held in Accelalox, and serves as a board member, owner, officer, or committee member of the American Association of Hip and Knee Surgeons and the Association of Bone and Joint Surgeons. Neither of the following authors nor any immediate family member has received anything of value from or has stock or stock options held in a commercial company or institution related directly or indirectly to the subject of this article: Dr. Hart and Dr. Kohrt. 
Division of Rheumatology and Clinical Immunology, Department of Medicine, University of Pittsburgh, Pittsburgh, PA

Dr. Mary I. O'Connor, MD, and

Department of Orthopedic Surgery, Mayo Clinic, Jacksonville, FL

\author{
Dr. Wendy Kohrt, PhD \\ Division of Geriatric Medicine, the University of Colorado School of Medicine, Denver, CO
}

Osteoarthritis (OA) is a leading cause of disability in the United States. It is the most common form of arthritis and afflicts $13.9 \%$ of adults aged $\geq 25$ years and 33.6\% (12.4 million) of those aged $>65$ years—an estimated 26.9 million persons in the United States. ${ }^{1}$ Studies sponsored by the Centers for Disease Control and Prevention and the National Institutes of Health have identified differences in the incidence and severity of OA between men and women, as well as between racial and ethnic groups. ${ }^{2,3}$

The burden of OA is highest among women and African-Americans, who disproportionately develop knee and hand but not hip OA. The disproportionate number of women in the aging US population is of clinical concern because of the more severe knee OA and its impact on quality of life and independence.

Based on these factors, there is a need for research focused on the effect sex differences have in the development and progression of OA as well as the impact on prevention and treatment strategies. However, most studies on the mechanisms underlying OA have not taken sex differences into account, whether in vitro cell culture or animal models were used. Although little is known about the mechanisms that contribute to disparities between men and women in disease incidence and severity, they likely involve mechanical and molecular events in the affected joint.

Diagnosis of knee OA is based on evidence of joint pain and/or reduced space between articulating bone surfaces as a result of thinning of the opposing articular cartilages. However, multiple tissues that compose the knee joint appear to be compromised by the disease, including subchondral bone, articular cartilage, the meniscus, the anterior cruciate ligament, the synovium, and synovial fluid. A change in any of these tissues can influence the distribution of load across the joint, with corresponding adaptations in the other tissues and, ultimately, the cartilages. Such patho-physiologic changes may exacerbate age-related physiologic changes in joint function attributable to genetic characteristics, age, sex, and health status, leading to greater cartilage damage.

To understand the expression of knee OA in males and females, it is important to view the knee as an organ rather than focusing only on the articular cartilage. Knee tissues are modulated by sex hormones during tissue development and throughout the life cycle in both males and females. Although menopause is associated with an increase in OA severity in women, systemic estrogen alone cannot explain the observed sex differences. Recent data, for example, show that sex-specific variations in the responses of chondrocytes to sex steroids are the result of differences in receptor number as well as mechanisms of hormone action. $^{4}$ 
In addition to increased prevalence of knee OA, women often have greater pain and more substantial reduction in function and quality of life than do men. ${ }^{5} \mathrm{OA}$ pain can be related to the sensory information that emerges from the knee joint. The pain does not always match the degree of injury, however, and can continue even after total joint arthroplasty. The neural and other mechanisms underlying these differences in pain between men and women with knee OA are unknown. By improving our understanding of the mechanisms responsible for sex differences in the perception of pain in OA, more effective and, possibly, sex-specific treatment strategies will emerge.

Although the adaptations that accompany advancing age may be a major factor in its etiology in older patients, early-onset OA is becoming more common. Women with physically active lifestyles, such as athletes and workers in occupations that involve exposure to traumatic injury or to mechanical stress, are more subject to early onset OA. Anterior cruciate ligament injuries are particularly problematic in 16- to 20-year-old females. Approximately $50 \%$ of these young women will progress to OA in 10 to 15 years. 6,7

The prevalence of obesity in children and young adults is escalating, and the impact of increased mechanical stress on the knee during bone growth and development is not yet fully understood. In addition, the role of sex differences, particularly hormonal regulation, and its influence on the onset and progression of OA is not yet known.

In summary, epidemiologic studies have established that sex differences exist in the incidence and severity of knee OA. Therapeutic approaches to the management of OA, particularly regenerative medicine strategies, have not yet taken these sex differences into consideration. Effective interventions, however, will require a better understanding of the mechanisms involved in the disease and its differential expression in men and women.

\section{References}

1. Felson DT, Lawrence RC, Dieppe PA, et al. Osteoarthritis: New insights. Part 1: The disease and its risk factors. Ann Intern Med. 2000; 133(8):635-646. [PubMed: 11033593]

2. O'Connor MI. Sex differences in osteoar-thritis of the hip and knee. J Am Acad Orthop Surg. 2007; 15(suppl 1):S22-S25. [PubMed: 17766785]

3. Srikanth VK, Fryer JL, Zhai G, Winzen-berg TM, Hosmer D, Jones G. A meta-analysis of sex differences prevalence, incidence and severity of osteoarthritis. Osteoarthritis Cartilage. 2005; 13(9):769-781. [PubMed: 15978850]

4. Kinney RC, Schwartz Z, Week K, Lotz MK, Boyan BD. Human articular chondrocytes exhibit sexual dimorphism in their responses to 17beta-estradiol. Osteoarthritis Cartilage. 2005; 13(4):330337. [PubMed: 15780646]

5. Zhang W, Nuki G, Moskowitz RW, et al. OARSI recommendations for the management of hip and knee osteoarthritis: Part III. Changes in evidence following systematic cumulative update of research published through January 2009. Osteoarthritis Cartilage. 2010; 18(4):476-499. [PubMed: 20170770]

6. Bien DP. Rationale and implementation of anterior cruciate ligament injury prevention warm-up programs in female athletes. J Strength Cond Res. 2011; 25(1):271-285. [PubMed: 21116195]

7. Brophy RH, Silvers HJ, Mandelbaum BR. Anterior cruciate ligament injuries: Etiology and prevention. Sports Med Arthrosc. 2010; 18(1):2-11. [PubMed: 20160623] 